\title{
Direct Integration of Government Funding and Family Support for Musculoskeletal Tumor Care in a Resource-Constrained Country
}

\author{
O.O. Adegbehingbe ${ }^{a} \quad$ A.L. Akinyoola ${ }^{a} \quad$ A.L. Ariyibi ${ }^{b} \quad$ L.M. Oginni ${ }^{a}$ \\ a Department of Orthopedic Surgery and Traumatology, Faculty of Clinical Sciences, Obafemi Awolowo University, \\ Ile-Ife, and ${ }^{b}$ Federal Medical Center, Department of Orthopedic Surgery and Traumatology, Ido Ekiti, Nigeria
}

\section{Key Words}

Bone cancer • Cancer care funding • Psychosocial impact • Poverty

\begin{abstract}
Background: Malignant musculoskeletal tumor (MMST) outcome reflects the level of supportive care provided. In Nigeria, the supportive care available to patients with MMST is limited by a lack of funding. Patients often present late, and receive only orthodox care as psychosocial care is not available. We evaluated the effect of direct incorporation of government funding and family support on MMST patient acceptance and completion of treatment. Methods: A 3-year prospective multicenter intention-to-treat study was undertaken in a tertiary care setting. The first step was a nonselective randomization of MMST patients into 2 groups, Wesley Guilds Hospital Firm A (WGHFA) and Wesley Guilds Hospital Firm B (WGHFB) using the computer software Excel. The control group was WGHFA, which consisted of patients who selffinanced their oncology care. The WGHFB patients' governments (local or state) provided funds according to medical reports and the patients had relatives who offered psychosocial support. The second step entailed treatment (surgery, chemotherapy and radiotherapy) and follow-up. Outcome measures were the interval between presentation at hospital and surgical intervention, acceptability of limb amputation,
\end{abstract}

completeness of treatment courses, duration of hospital stay and mortality. The reproducibility of the methodology was reappraised at the Federal Medical Center. Kruskal-Wallis analysis was used, and an alpha error of $<0.05$ at a $\mathrm{Cl}$ of $95 \%$ was taken to be significant. Results: A total of 112 cases of MMST were managed during the study period. Seventy-one (63.4\%; 37 WGHFA; 34 WGHFB) met the inclusion criteria. Age, sex, tribe, religion, comorbid factors and mean weekly income were not significant factors influencing improved MMST care among the WGHFB patients. 32 WGHFB versus 7 WGHFA patients accepted the treatment plan. The mean duration of hospital stay before surgery ( $p<0.001)$, discharge against medical advice ( $p<0.000)$, limb salvage ( 21 vs. $2, p<$ 0.001), limb amputation (3 vs. 12, $\mathrm{p}<0.05,95 \% \mathrm{Cl} 8.3-37.9$ ), completeness of treatment ( 33 vs. $7, p<0.05$ ), mean duration of hospital stay, in days (23 vs. $39, \mathrm{p}<0.05)$ and mortality at 1-year follow-up (13 vs. $28, \mathrm{p}<0.02$ ) were significant. Conclusion: The cost of cancer care is a challenge for patients with MMST in a resource-constrained country such as Nigeria. Direct integration of the government and family into MMST care will serve as a link between the cancer patient and the source of funds. It raises the possibility of an effective psychosocial approach to improve patient outcome through enhanced treatment acceptability and completion, and so reduce morbidity and short-term mortality.

Copyright ๑ 2009 S. Karger AG, Basel

Dr. O.O. Adegbehingbe

Obafemi Awolowo University, Department of Orthopedic Surgery and Traumatology Ile-Ife, Osun State (Nigeria)

Tel. +234703136 722, Fax +23436230141

E-Mail olayinkaadegbehingbe@yahoo.co.uk

\begin{tabular}{ll}
\hline KARGER & ( 2009 S. Karger AG, Basel \\
Fax +41613061234 & $0030-2414 / 09 / 0766-0398 \$ 26.00 / 0$ \\
$\begin{array}{l}\text { E-Mail karger@karger.ch } \\
\text { www.karger.com }\end{array}$ & $\begin{array}{l}\text { Accessible online at: } \\
\text { www.karger.com/ocl }\end{array}$
\end{tabular}




\section{Introduction}

Across the world, about 25 million people are living with cancer [1]. It is the second leading cause of death worldwide and the third leading cause of death in developing countries $[1,2]$. The disease burden has shifted to low- and middle-income countries, where more than half of all new cancer cases occur today [1]. Health economists predict that cancer could become a major impediment to socioeconomic development in low-income and economically emerging nations [1]. There is an increasing recognition within the oncology community of the immense burden associated with cancer among citizens of developing countries [2].

Treatment challenges in resource-constrained countries include lack of funding and inefficient use of the funds that are available for oncology prevention, early detection and treatment. Public fund diversion and failure to include musculoskeletal cancer care in the national health insurance scheme are also likely attributes [3]. Practitioners in such countries also face technological difficulties, political instability, an inconsistent health policy, poverty, corruption, and ignorance of cancer morbidity and mortality [4]. Treatment problems experienced by malignant musculoskeletal tumor (MMST) patients are related to low levels of awareness, misinformation through negative cultural practices and religious doctrine, family conflict, misdirection as to where adequate care could be received and the high costs of treatment. There is no adequate psychosocial support available. The consequences of the aforementioned factors are late presentation, restriction to orthodox care and high mortality after confirmation of diagnosis $[3,4]$.

The musculoskeletal tumor outcome reflects the level of supportive care [5]. The supportive care available to patients with MMST can be limited by a lack of funds. In Nigeria, a resource-constrained country, musculoskeletal cancer patients often have difficulties accessing available treatment. Direct integration of government funding and family support for musculoskeletal tumor care in a resource-constrained country has not previously been investigated. The aim of this study was to evaluate the effect of direct incorporation of government/health-policy makers and patients' relatives by a musculoskeletal oncologist on MMST patients' acceptance and completion of treatment. The research hypothesis was that effective psychosocial support has a direct effect on the MMST patient's outcome.

\section{Patients and Methods}

A 3-year prospective multicenter intention-to-treat study was undertaken between January 2005 and December 2007. The setting was a tertiary health care center based at the teaching hospital complex of Obafemi Awolowo University in Ile-Ife, Osun State, western Nigeria. It includes the Wesley Guilds and the Ife State Hospitals. The institution's medical research and ethics committee approved the study protocol. The patients gave informed written consent and video documentation of family conflicts related to cancer care funding.

The first step was a nonselective randomization of MMST patients into 2 groups (WGHFA and WGHFB) using the computer software Excel. The patients who served as control subjects were those in WGHFA, and they financed their oncology care personally. Patients in WGHFB formed the experimental group. Their governments (local and state) were approached to directly provide funds for each patient's cancer care. The family and relatives offered psychosocial support. The relatives of each patient sent a written medical report containing the patient's clinical-radiological features and histological diagnosis to their respective local government chairman and state governor via the commissioner for health. The individual local or state medical intervention boards reviewed each MMST patient's case. A letter was written by the involved health management board to the orthopedic oncologist requesting the complete treatment plan and detailed estimate of cancer care. The funding approved for each patient was paid into the central hospital account. The cancer patient was given an account code into which all investigation, treatment and follow-up costs were channeled.

The second step entailed treatment (surgery, chemotherapy and radiotherapy) and follow-up. All enrolled subjects completed a sociodemographic questionnaire to elicit biodata and assess their weekly income (many people are not on a monthly payroll). The General Health Questionnaire (GHQ 30), the Sate-Trait Anxiety Inventory (STAIX-1) and the Zung Self-Rating Depression Inventory were completed for each enrolled subject. The outcome measures were the interval between the patient's hospital presentation and surgical intervention (in days), acceptability of limb amputation, completeness of treatment courses, duration of hospital stay after surgery (in days) and mortality. The reproducibility of the methodology was reappraised at the Federal Medical Center in Ido Ekiti, Ekiti State. The study limitations were inadequate follow-up period, no homogenous MMST and only limited cases available for the study.

\section{Statistical Analyses}

All analyses were performed on the basis of the intention-totreat cohort, defined as all MMST patients who took at least 1 form of cancer treatment. Data were analyzed by using the Statistical Package for Social Sciences (SPSS) version 15.0 for Windows. The comparability of patients in the 2 treatment groups was determined from the demographic data and baseline hemodynamic values. The Kruskal-Wallis analysis was used for the generated data. Changes in the mean weekly income of the group and mean duration of hospital stay before and after surgery were evaluated using 2-way ANOVA. At a CI of $95 \%$ p $<0.05$ was taken to be significant. 


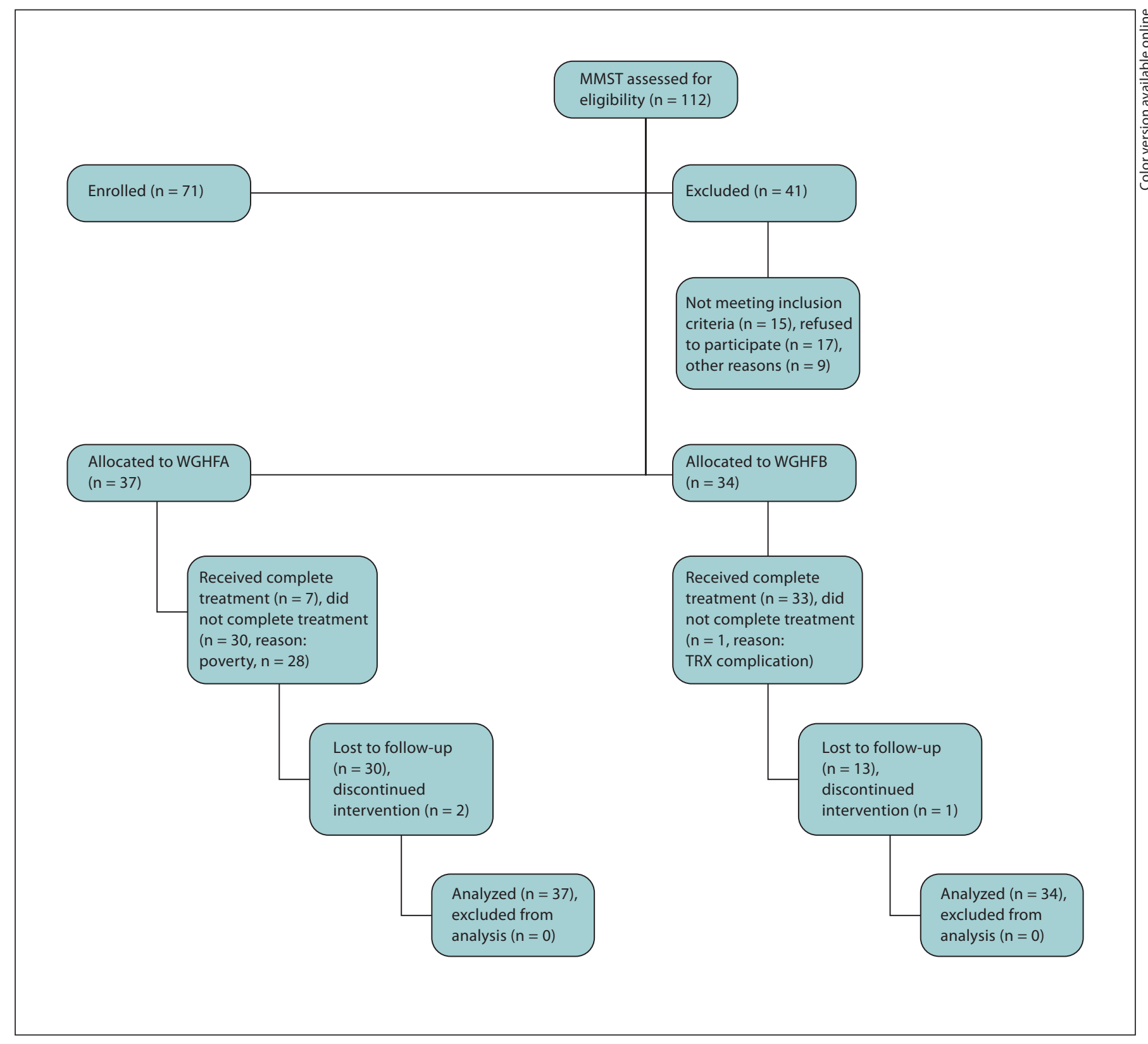

Fig. 1. The eligibility flowchart of MMST patients evaluated for DIGAFA.

\section{Results}

A total of 112 cases of MMST were managed during the study period. Seventy-one $(63.4 \%, 37$ WGHFA and 34 WGHFB) met the inclusion criteria, as shown in figure 1 . Age, sex, tribe, religion and mean weekly income were not significant factors influencing improved MMST care among the WGHFB patients, as illustrated in table 1 . The patterns of MMST included 42 (59.2\%) osteosarcoma, 10 (14.1\%) squamous cell carcinoma, 7 (9.9\%) giant cell tumor, 5 (7.0\%) synovial sarcoma, 3 (4.2\%) neurofibromyosarcoma, 2 (2.8\%) Kaposi's sarcoma and 2 (2.8\%) rhabdomyosarcoma, as shown in figure 2.

The difficulties encountered were delay in the release of funds ( $n=9,26.5 \%)$, patients attempting to inflate the cancer cost estimates sent to the government $(n=4$, 
Table 1. Demography data of MMST evaluated for study

\begin{tabular}{lcc}
\hline Biodata & WGHFA $(\mathrm{n}=37)$ & WGHFB $(\mathrm{n}=34)$ \\
\hline Age, years & $5(13.5)$ & $3(8.8)$ \\
$11-20$ & $24(64.8)$ & $27(79.4)$ \\
$21-30$ & $1(2.7)$ & $3(8.8)$ \\
$31-40$ & $3(8.1)$ & $1(2.9)$ \\
$41-50$ & $4(10.8)$ & $0(0.0)$ \\
$51-60$ & $23(62.2)$ & $19(55.9)$ \\
\hline Sex & $14(37.8)$ & $15(44.1)$ \\
Male & $37(100.0)$ & $34(100.0)$ \\
Female & & \\
\hline Tribe (nationality) & $29(78.4)$ & $26(76.5)$ \\
Yoruba (Nigerian) & $1(2.7)$ & $3(14.7)$ \\
\hline Religion & $27.5($ range 22.0-40) & $28.0($ range 20.9-38.0) \\
Christian & & \\
Muslim & $0(0.0)$ & $1(2.9)$ \\
Other & $8(21.6)$ & $3(8.8)$ \\
\hline Mean weekly income, USD ${ }^{1}$ & $1(2.7)$ & $0.0)$ \\
\hline Comorbidity & 118 NGN). & \\
Diabetic mellitus & & \\
Heart disease & & \\
HIV/AIDS & & \\
\hline Except where stated otherwise, values in parentheses are percentages. & \\
\hline Converted from Nigerian Naira $(1 \mathrm{USD}$ & \\
\hline
\end{tabular}

$11.7 \%)$ and corrupt practices of a health ministry paymaster $(n=1,2.9 \%)$ who took a percentage from the patient's relatives. Delays in releasing funds were related to political instability affecting the officials who approved the financial support. Six patients were affected because the health-policy maker was changed during a cabinet reshuffle of their state government. Three patients lost their financial support because their local government chairmen were removed from their positions by an election tribunal.

Table 2 shows the effects of direct integration of government funding and family support on musculoskeletal tumor care.

The mean personal financial contribution made by each WGHFA patient was about USD 840, as compared to about USD 100 for each WGHFB patient. The total direct contribution to cancer care from local and state governments was USD 48,000 and USD 69,000, respectively. A total of 32 WGHFB versus 7 WGHFA patients accepted the treatment plan. Significant differences between groups were found in mean duration of hospital stay be-

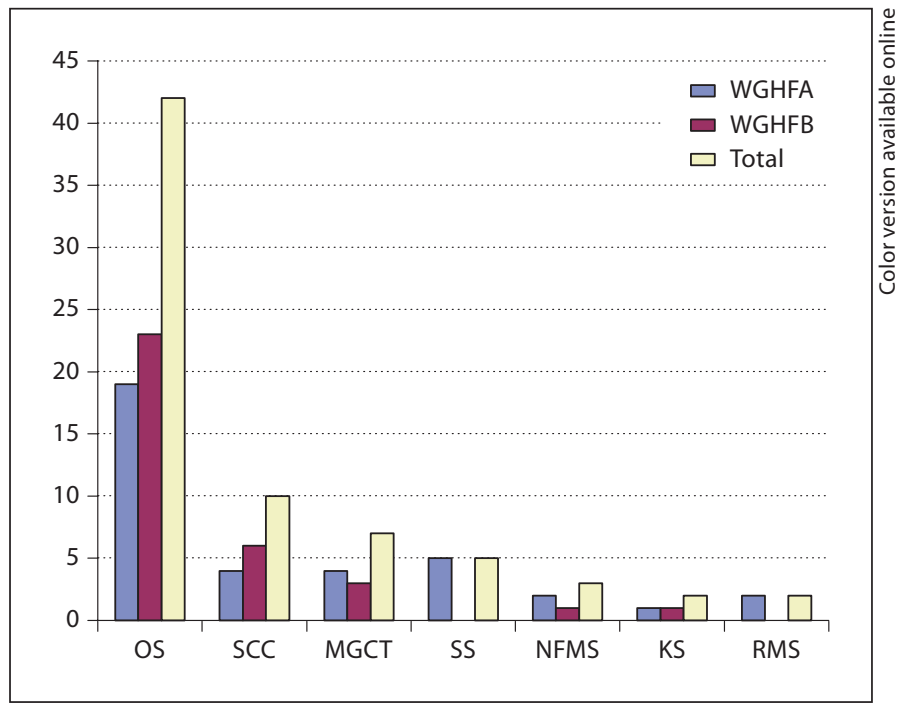

Fig. 2. Pattern of MMST evaluated for direct integration of government funding and family support. OS = Osteosarcoma; SCC $=$ squamous cell sarcoma; MGCT = malignant giant cell tumor; SS = synovial sarcoma; NFMS = neurofibromyosarcoma; KS = Kaposi's sarcoma; RMS = rhabdomyosarcoma. 
Table 2. Effects of direct integration of government funding and family support on musculoskeletal tumor care

\begin{tabular}{|c|c|c|}
\hline Biodata & WGHFA $(n=37)$ & WGHFB $(\mathrm{n}=34)$ \\
\hline \multicolumn{3}{|l|}{ Accept the treatment plan (surgery, chemotherapy, } \\
\hline Mean duration of hospital stay before surgery, days & 34 (range $23-61$ ) & 11 (range 6-19) \\
\hline Discharge against medical advice & $26(70.3 \%)$ & $1(2.9)$ \\
\hline Limb salvage & $2(5.4 \%)$ & $21(61.7)$ \\
\hline Limb amputation & $3(8.1 \%)$ & $12(32.3)$ \\
\hline Completed the treatment plan & $7(18.9 \%)$ & $33(97.0)$ \\
\hline Mean duration of hospital stay after surgery, days & 39 (range $28-56$ ) & 23 (range: $21-29$ ) \\
\hline Mortality at 1 -year follow-up & $28(75.6 \%)$ & $13(38.2)$ \\
\hline \multicolumn{3}{|l|}{ Mean financial contribution, USD ${ }^{1}$} \\
\hline Personal & 838.9 (range $358-1,439)$ & 97.5 (range $52-147$ ) \\
\hline Local government & 0.0 & $48,083.8$ (range $424-2,783$ ) \\
\hline State government & 0.0 & $69,006.4$ (range $1,102-5,554)$ \\
\hline \multicolumn{3}{|l|}{ Psychosocial factors } \\
\hline Anxiety & $83.8 \%$ & $14.7 \%$ \\
\hline Depression & $78.4 \%$ & $11.8 \%$ \\
\hline
\end{tabular}

fore surgery $(\mathrm{p}=0.001)$, discharge against medical advice $(\mathrm{p}=0.000)$, limb salvage (21 WGHFB vs. 2 WGHFA, $\mathrm{p}=$ 0.001 ), limb amputation acceptability ( 3 vs. $12, \mathrm{p}<0.05$, 95\% CI 8.3-37.9), completeness of treatment (33 vs. 7 , $\mathrm{p}=0.000)$, the mean duration of hospital stay after treatment ( 23 vs. 39 days, $\mathrm{p}<0.05)$ and mortality at 1 -year follow-up (13 vs. $28, \mathrm{p}=0.02$ ). Poverty was a contributing factor to treatment delay in patients in the WGHFA group, with lack of funds being associated with discharge against medical advice. Among the WGHFB patients, 7 (20.6\%) experienced treatment delay related to lack of hospital bed space for immediate admission, while 5 (14.7\%) were affected by a lack of theater space for surgery due to renovation. One patient in the WGHFB group took discharge against medical advice after the relative who stayed with her in hospital had a change in her employment.

The psychological morbidity was high among the WGHFA patients with $87.7 \%$ having a GHQ 30 score $>5$ compared to $23.4 \%$ of the WGHFB patients. Anxiety and depressive symptoms were high, as shown in table 2 . The correlation analysis showed that there was significant correlation between anxiety and government funding and family support, while the depressive symptoms correlated with completeness of the treatment plan. This study showed our original hypothesis (that effective psychosocial support has a direct effect on outcome in MMST patients) was true. The study methodology is easily reproducibile. The clinical implications of this study are that integration of funding and family support affect morbidity and mortality among MMST patients in a resource-constrained country (Nigeria).

\section{Discussion}

An estimated 650,000 new cases of cancer occurred in Africa in 2002 (530,000 of which were in sub-Saharan Africa, which includes Nigeria, the setting for this study). There were 311,000 cases in males and 339,000 in females [6]. Cancer kills more people than AIDS, tuberculosis and malaria combined and will become the world's single leading cause of death by 2010 [7]. It has been estimated that by 2030 more than 12 million people could die from this disease every year. About $80 \%$ of these deaths are likely to occur in low-income and middle-income countries, where resources available for cancer control are restricted or nonexistent [7].

Direct integration of government funding and family support into MMST care was an innovative idea. This concept arose from repeated frustrations and failures experienced by poverty-stricken MMST patients to get their respective government institutions to budget funds for oncology care. Policy makers and health law implement- 
ers in resource-constrained countries are far away from the populations they serve, the majority of which live in rural and suburban areas. Cancer patients often cannot make their voices heard at the heart of government, and there is no health care or welfare for individuals with MMST in countries with limited financial resources. The high MMST morbidity and mortality rates seen in the WGHFA patients had not been recognized before this study by the health policy formulators and implementers in the local and state governments in Nigeria. To date, international grants and nongovernment organization funding for cancer research and treatment in resourceconstrained countries have been managed or manipulated by government bodies without any direct or indirect improvement for MMST patients. The oncologists and patients involved are left to struggle against cancer-imposed poverty without the funds to access available cancer care. This is the central problem Nigeria faces in cancer control and explains why Nigerian MMST patients have a much poorer prognosis than their counterparts in developed countries. Overcoming the high MMST morbidity and mortality will require the direct involvement of orthopedic oncologists in resource-constrained countries.

The provision of services for the prevention and treatment of MMST has not been a priority for African governments and development agencies, which have focused on the more common health issues, such as communicable diseases and maternal and child mortality, which have been largely solved in the developed world $[8,9]$. The community health centers initiative was launched in 1965 as part of the Lyndon B. Johnson administration's War of Poverty program. It was largely brought about by Dr. Jack Geiger who observed the South African movement that created facilities where poor workers could receive both public health services and medical care [8]. Geiger's primary policy goals were to combine disparate models and remove financial barriers to access. Reflecting this 'power to the people' philosophy, the funding mechanism for MMST consists of state government grants and local government funds that could bypass ministry/council officials and flow directly to the patient's orthodox care center. Integrating funding and family support enables relatives and medical social workers to provide adequate psychosocial support for individual cancer patients. The default rate of the treatment plan, the noncompliant use of chemotherapy and radiotherapy together with refusal of limb amputation were reduced. Poverty and inaccessibility of health services were not related to a particular religious group or gender. The integrated approach has brought out the impact of socioeconomic status on MMST patients and the unique cultural perspectives that could strongly influence how cancer care conditions might be approached in a resource-constrained country.

The low levels of awareness of MMST, patient poverty and lack of funding for treatment are the main problems for the care of those with cancer. A specific workable method of funding MMST care in resource-constrained countries is not uniformly available or implemented. Dr. Margaret Chan, Director-General of the World Health Organization, alluded to the magnitude of the problem at the World Cancer Congress in Geneva in 2008. She said: 'Diseases like cancer are a leading cause of so-called catastrophic health expenditure. This is especially true in low- and middle-income countries, where most people rely on out-of-pocket payments for health care' [1]. Also she went further, saying that 'for cancer, out-of-pocket payment is a double-edged sword. It discourages people from seeking treatment early, when the chances of cure are greatest, and it deepens household and community poverty' [1]. These statements succinctly explain the reasons for late presentation, discharge against medical advice and relying on orthodox care seen among WGHFA patients in our study. The integrated approach appeared to reduce psychological symptoms among WGHFB patients, indicating the importance of the social and emotional support received by these patients.

In Nigeria, MMST patients face poverty because of the failure of governments to address inequalities in national wealth distribution and administrative corruption. If there was less financial inequality many cases of malignant musculoskeletal cancer could be prevented, detected early or even cured. For MMST control in low- and middle-income countries, breakthroughs might not come in the form of spectacular new drugs for cure, but immediate improvements can occur by integrating government funding and family support. This shows how existing tools can be adapted to improve cancer patient care and obtain a good psychosocial outcome in settings where resources are scarce.

\section{Conclusion}

In resource-constrained countries such as Nigeria, the out-of-pocket costs of cancer care prevent many MMST patients from seeking, receiving or completing appropriate treatment courses. Direct integration of government funding and family support into musculoskeletal tumor care will serve as a link between the cancer 
patient and the direct source of funding. This integrated approach improves the patient's outcome and enhances the acceptability and completion of treatment. It also reduces morbidity and short-term mortality of MMST patients.

\section{References}

1 Daily News, August 28, 2008. UICC World Cancer Congress 2008, Geneva, Switzerland.www.uicc.org/templates/uicc/pdf/congress08/dailynews_28_web.pdf (accessed January 2, 2009).

-2 Markman M: Reducing the worldwide burden of cancer. Oncology 2007;73:1.

3 Adegbehingbe OO, Oginni LM, Adelusola KA, Olabanji JK: Limb salvage surgery in malignant musculoskeletal tumor: current trends in a developing country without customized end prostheses. Tanzanian Journal of Health Research, 2008, in press.

\section{Acknowledgements}

We thank the state governors, commissioners for health (especially Dr. Femi Thomas of Ekiti State and Dr. R. Mimiko of Ondo State) and the local government chairmen in Ekiti State, Ondo State, Osun State and Oyo State of Nigeria. This paper was presented at the 2008 World Cancer Congress in Geneva, Switzerland.
4 Adegbehingbe OO, Ojo SO, Owa OT, Olayade KO, Adegbenjo TA: Awareness of Bone Tumor in Nigeria. Ghana Medical Journal, 2008 , in press.

5 Gloeckler LA, Reichman MA, Lewis DR, Hankey BF, Edwards BK: Cancer survival and incidence from the surveillance, epidemiology and end results (SEER) program. Oncologist 2003;8:54-55.
6 Parkin DM, Sitas F, Chirenje M, Stein L, Abratt R, Wabinga $\mathrm{H}$ : Cancer in indigenous Africans: burden, distribution and trends. Part I. Lancet Oncol 2008;9:683-692.

7 Seffrin JR: Cancer control as a human right. Lancet Oncol 2008;9:409-411.

$>8$ Yamada T: In search of new ideas for global health. N Engl J Med 2008;358:1324-1325.

9 World Health Organization: The World Health Report 2004: Changing History. Geneva, WHO, 2004. 